\title{
THE INVARIANT SUBSTITUTIONS UNDER A SUBSTITUTION GROUP.
}

\author{
BY PROFESSOR G. A. MILLER.
}

(Read before the American Mathematical Society, September 6, 1907.)

WE begin with the case where the substitution group $G$ is transitive and of degree $n$. If the subgroup $G_{1}$ composed of all the substitutions of $G$ which omit a given letter is of degree $n-1$, there is no substitution which involves any of the letters contained in the substitutions of $G$ and is also commutative with every substitution of $G$. In considering substitutions which are commutative with every substitution of $G$ we shall confine ourselves to those which involve no letters that are not also contained in $G$, since every substitution which does not involve any of the letters of $G$ is clearly commutative with every substitution of $G$. Hence we may say: when the degree of $G_{1}$ is $n-1$, identity is the only substitution which is commutative with every substitution of $G$.

When $G_{1}$ is identity it is well known that there are exactly $n$ substitutions which are commutative with every substitution of $G$, and that these constitute a group which is simply isomorphic with $G$, known as the associate of $G$, whenever $G$ is non-abelian.* It remains to consider the case where $G_{1}$ is of degree $n-\alpha(1<\alpha<n)$. All the substitutions which transform $G_{1}$ into itself constitute a subgroup of order $\alpha g \div n, g$ being the order of $G$. This subgroup may clearly be constructed by establishing a $\left(g_{1}, 1\right)$ isomorphism between a group of order $\alpha g \div n$ and a regular group of order $\alpha, g_{1}$ being the order of $G_{1}$. If this regular group is abelian it is transformed into $n \div \alpha$ distinct regular groups by the substitutions of $G$ and a simple isomorphism between these groups which is so constructed that all the conjugates of a given substitution correspond will be composed of all the substitutions which are commutative with every substitution of $G$. If the regular group of order $\alpha$ is non-abelian its associate is transformed in the manner stated. Hence we have the theorems: The necessary and sufficient condition that there are substitutions besides identity which are com-

\footnotetext{
* Quar. Jour. of Math. vol. 28 (1896), p. 249. When $G$ is abelian it is
} self-associate. 
mutative with every substitution of a transitive group of degree $n$ is that the subgroup which is composed of all its substitutions omitting a given letter is of a degree lower than $n-1$. If the degree of this subgroup is $n-\alpha$, there are exactly a substitutions which are separately commutative with every substitution of the transitive group, where a may be any divisor of $n$ but can have no other value.

This theorem may be regarded as a generalization of the theorem that there are just $n$ substitutions which are commutative with every substitution of a regular group. It is also directly applicable to intransitive groups, since the total number of substitutions which are commutative with every substitution of such a group and do not interchange any of its systems of intransitivity is composed of the direct product of those which are commutative with all the substitutions of its transitive constituents. While the number of substitutions which are commutative with every substitution of a transitive group is always a divisor of the degree of the group, the number of those which have this property and do not permute the systems is a divisor of the product of the partitions of this degree with respect to addition when the group is intransitive.* Although every transitive group which is such that there are substitutions which are commutative with every one of its substitutions is necessarily imprimitive, $\dagger$ it is not true that every imprimitive group has this property, since there are imprimitive groups of degree $n$ which involve subgroups of degree $n-1$. The preceding developments have close contact with those given on page 124 of Maillet's Thèse de Doctorat, 1892, and by Burnside, "Theory of groups of finite order," 1897, page 217 .

The $\alpha$ substitutions which are commutative with every substitution of $G$ when $G_{1}$ is of degree $n-\alpha$ are not necessarily contained in $G$ whenever $\alpha>1$. They are however contained in the largest group of degree $n$ which transforms $G$ into itself. We thus arrive at a generalization of a property of the holomorph of a group, which may be stated as follows : The largest group of degree $n$ which transforms a transitive group of degree

* The addends are clearly the degrees of the systems of intransitivity and the total number of substitutions which are commutative with every substitution of an intransitive group is a divisor of the product of the degrees of its transitive constituents and the number of ways in which these constituents may be transformed into each other.

$\uparrow$ Except the cyclic group of prime order. 
$n$ into itself contains $\alpha$ substitutions which are commutative with every substitution of this transitive group when its subgroup composed of all the substitutions which omit a given letter is of degree $n-\alpha$. In the case of the holomorph of $G, \alpha=n$.

UNIVERSITY OF ILLINOIS, July, 1907.

\section{SHORTER NOTICES.}

Leçons d'Algèbre et d'Analyse à l'Usage des Elèves des Classes de Mathématiques spéciales. Par Jules Tannery. Tome Premier. Paris, Gauthier-Villars, 1906. 423 + vii pp.

This text is prepared for students of the classe de mathématiques spéciales of the French lycées, in which boys of 18 or 19 prepare themselves for admission to the Ecoles normale and polytechnique. In so doing, they go over an amount of mathematics which seems overwhelming.* If the contents of the volume under review are even approximately an indication of what is taught successfully in one year, in any one of several subjects pursued by classes of boys of ordinary ability, then we have indeed much to learn from the teachers in the French lycées. But the contrast is less pronounced when we consider the difference in aim. The French adapt their courses to the abilities of the 2 per cent. or 5 per cent. who are the most gifted ; Americans adapt their work to the capacity of the average boy. In the French lycées the 95 per cent. or 98 per cent. who fail in the first trial repeat the course during a second year, whereupon about 25 per cent. succeed. Less than half are said to succeed even after the third trial. Much can be said for and much against such a system of highly competitive examinations.

The first chapter of Tannery's book, covering 58 pages, contains a detailed exposition of irrational numbers. Adopting an easy conversational style, the author makes the subject very clear, except that, in a few instances, it is not stated from the start what the underlying assumptions are. Whenever such doubt lurks in the mind of the reader, he will find that the

* Bulletin, vol. 6 (1900), p. 233. 